\title{
COMPLEMENTING CLASSROOM EXPERIENCE WITH STUDENT-RUN WORKSHOPS
}

\author{
Dario Schor, Troy Denton, Matthew Sebastian, Matthew Woelk, Frank Serafin, Craig Nemeth, \\ Pawel Glowacki, Kiral Poon, Kane Anderson, Greg Linton, Arash Fazel Darbandi, \\ Witold Kinsner, Allan McKay, Kenneth Biegun, and Udaya Annakkage \\ Dept. of Electrical \& Computer Engineering, Faculty of Engineering \\ University of Manitoba, Winnipeg, MB, Canada R3T 5V6 \\ \{dschor|kinsner\}@ece.umanitoba.ca
}

\begin{abstract}
Professors, instructors and technical staff at various universities often face difficult questions regarding the tools and hands-on skills taught in the curriculum during a limited laboratory time. Therefore, to complement the classroom experience, students in Electrical and Computer Engineering at the University of Manitoba have developed and delivered a number of workshops on topics that have a direct impact in the classroom and job prospects, while encouraging independent learning and experimentation to foster lifelong learning skills. This paper describes the organization and examples from organizing more than 20 workshops that reached excess of 250 undergraduate students, graduate students, high school students, high school teachers, recent graduates, industry professionals, and hobbyist.
\end{abstract}

Keywords: Student-run workshops; lifelong learning.

\section{INTRODUCTION}

In the busy engineering curriculum, only a small subset of tools and hands-on skills are often selected for teaching in a laboratory setting on the basis of their educational merit, transferable skills, and applicability in industry. This can be further complicated for specialized activities where the learning curve for the tool far exceeds the available class time forcing professors must trade-off a shallow exposure to industry tools versus an in-depth understanding of simplified tools that perform similar tasks. In order to complement the classroom experience, students in Electrical and Computer Engineering at the University of Manitoba (UofM) with the support of professors and staff from the Department are leading hands-on workshops on a variety of topics.

Since September 2010, students from the UofM IEEE Student Branch (UMIEEE), UofM Amateur Radio Society (UMARS), and UofM Space Applications and Technology Society (UMSATS) have conducted more than a twenty workshops in topics including LaTeX,
Matlab, Simulink, C programming, amateur radio, orbital mechanics, and embedded systems [1][2]. The topics are selected to match students interests, required skills for courses, and to cover useful tools for capstone projects and graduate thesis. For each workshop, one or more students knowledgeable in the subject prepare the material, while others assist with the administrative tasks and logistics. Each event is advertised with a list of topics to be covered, recommended pre-requisite knowledge, and a target audience (i.e., students taking or about to take specific courses). Guest speakers are invited if a topic is deemed important, but no student instructor has enough depth to deliver the content. Take-home materials and in some cases hardware are provided to all participants to continue their experiments on their own.

In addition to the acquired knowledge for students attending the workshops, there are many benefits for all the other stakeholders involved. The presenters get a chance to prepare content such as slides, handouts, sample code, and schematics for the participants for different audiences. This experience enhances the communication skills for all presenters and tests their own understanding of the subject. Novice students assisting with the administrative tasks for the workshops get experience in running events and acquire skills to run some of the activities themselves in the future. The student groups benefit through activities, training of members for various projects, and exposure leading to recruitment. Many students have joined the groups after attending the workshops to get discounted prices, advanced information about upcoming events with a chance for an early registration, or to improve and deliver some of the existing workshops. Furthermore, the activities serve both an academic and organizational purpose for the department. Students attending the workshops get additional skills to aid in their education and beyond, thus motivating them as they see links and expansions from the material covered in class to other areas of interest. The workshops are also used for outreach [2][3] and to strengthen the partnership with industry. A number of students from high schools, other post-secondary 
institutions, and students from other departments at the University of Manitoba have attended the activities and been encouraged to join the Faculty of Engineering, while some professionals attended the more advanced workshops as part of their professional development required by the local Association of Professional Engineers.

This paper presents an overview of a typical workshop, lessons learned from the students leading the activities, and highlights of some of the benefits and outcomes experience at the University of Manitoba.

\section{PLANNING WORKSHOPS}

Student-run workshops require extensive planning and collaboration from various groups. This section describes the sequence of events from the selection of the topic through the delivery of the workshop.

\subsection{Selecting Topics Criteria}

The topics for workshops are selected based on three primary criteria aimed at both complementing the classroom experience and exposing students to new fields, tools, and techniques.

Complementary Classroom Needs: This criterion addresses various techniques, tools, and topics related to courses that students are expected to learn on their own. For example, although the bare minimum Matlab programming techniques are taught in most programs, upper-level courses expect students to solve advanced problems. The workshops aim at providing a strong foundation of debugging techniques with an emphasis on matrix-based operations to optimize the performance of Matlab algorithms. Other examples of topics include Simulink, Electric Circuit Analysis, C programming, and Embedded Systems.

State-of-the-art Hands-on Needs: This criterion addresses tools and techniques that build on classroom knowledge for a more comprehensive intuitive understanding of a particular subject. For example, the students participating in the Canadian Satellite Design Challenge at the University of Manitoba identified a need for learning about orbital mechanics, so the student group organized a workshop on Orbital Mechanics using STK. This provided them with hands-on experience using the state-of-the-art tools for orbital mechanics. Other topics included the user of Arduinos for embedded systems, LaTeX tools, Capacitive Touch tools, and Linux tools.

Current Topics: This criterion allow students to explore topics of today, not covered in the classroom yet. These topics rely on current trends and developments internal to the University of Manitoba (e.g., amateur radio) and topics external to our local community (e.g., Arduinos).

\subsection{Defining Learning Objectives}

Prior to defining the learning objectives for a workshop, the organizers first select the type of workshop (exposure vs. specific) and identify the target audience. Some of the workshops are aimed at providing some exposure to a topic that encourages students to experiment on their own. For example, the LaTeX workshops possessed a large amount of information in a short amount of time that gave attendees an overview of the power of the typecasting language through many examples. Following the workshop, the attendees used the examples to build their own documents and apply the skills more thoroughly. On the other hand, some workshops were designed to refine specific skills such as the Advanced Embedded Systems tutorials aimed at interfacing different types of components to a microprocessor.

The audience for the workshop is of critical importance. The first Embedded Systems Workshop organized by the student groups entertained a large audience ranging from first year students through graduate students. This made it very difficult to define the parameters for the workshop. Learning from that experience allowed the group to define clear audiences with specific pre-requisite knowledge for each workshop. Thus, the subsequent Embedded Systems Workshops were divided into beginners, intermediate, and advanced students.

Having identified the high level objectives and the audience, the instructors for the workshop defined 3-5 specific learning objectives. These objectives would describe the key outcomes from the workshop such as, "after completing the workshop, students should be able to configure and read an analog port on the MSP430 microprocessor." The objectives were explicitly stated at the beginning of each workshop and checked at the conclusion of the workshop by prompting the attendees on what they learned during the workshop.

\subsection{Logistics \& Equipment}

The logistics for a workshop are planned in collaboration with the Department of Electrical and Computer Engineering. The organizers book the room and equipment that will be borrowed from the department.

Depending on the workshop duration and equipment, the organizers set a cost for the workshop. These are not designed to make profit, but rather aim to cover all expenses. Most workshops over 2 hours long offer some refreshments. In some cases (e.g., embedded systems) the attendees have the option to purchase the embedded processor (MSP430 LaunchPad [4] or Arduino UNO [5]). In the case where parts were sold to students, pre-orders are used to keep inventory to a bare minimum. 
Having the logistics in place, posters, emails, websites, and newsletters are used to advertise the workshop. The advertising includes the date, time, cost, registration procedures, abstract on the event including key objectives, and the recommended audience.

\subsection{Developing Materials}

The student instructors for the workshop are responsible for preparing slides to present the material and examples for hands-on exercises. All materials are made available online prior to the workshop so that all attendees can use them and advance through portions of the workshop at their own pace.

Most of the work goes into preparing exercises. In some cases, this involves sample code, schematics, sample simulations, or other components. The difficulty in this process is to identify and anticipate possible problems, test the output, and be familiar with proper techniques to debug and fix the problems. This preparation takes many hours, but serves a critical role when delivering the workshop and being able to suggest proper techniques to detect and resolve problems.

\subsection{Delivering Workshops}

At least one student-instructor delivers the main content for the workshop, while a group of students assist answering questions and provide logistical support (i.e., handling registrations and ordering refreshments). The assistants help ensure that the workshop keeps running as they are able to sit down with students falling behind so that the instructor can continue to deliver the main content at a pace that satisfies the majority of the group.

The actual delivery starts with a concise theoretical background on the topic being covered that refers students to other available sources of information. This also reinforces links between the hands-on activities and topics covered in other courses thus helping students pick electives or understand linkages with various topics covered.

Although the workshop material assumes a specific audience, it is also made relevant to their work experience. Consequently, instructors often prompt attendees about projects they are working on so that they can use a combination of verbal examples and descriptions of how the workshop exercises link to their work.

\subsection{Additional Logistical Activities}

In organizing the workshops, the instructors learned a number of lessons that include the following:

- Talk to the local Engineering Association (in this case the Association of Professional Engineers and
Geoscientists of Manitoba) to recognize the workshops are professional development. This encourages industry to attend and thus provides valuable networking opportunities within the workshop.

- Ask a few short questions on demographics in the online registration form to help the instructors customize the material for the audience.

- Limit workshops to 25 attendees. Larger groups are doable with many assistants, but it is harder to keep the group moving at a reasonable pace.

- Plan to arrive 1 hour prior to the workshop for setup and stay at least 2 hours after the workshop to answer questions and cleanup.

- $\quad$ Try to ensure that all participants complete the work before they leave. This may involve spending additional time with them or inviting them to one of the student groups later in the week to get additional help. The effort helps in recruiting and fostering the community atmosphere.

- Although most of the software used in the workshops (except for STK [6]) is open source, it is better to work in a laboratory with tools pre-installed on all workstations. Student laptops present many unexpected challenges that can affect the schedule for a workshop and discourage attendees.

\section{EXAMPLES OF WORKSHOPS AT THE UNIVERSITY OF MANITOBA}

The following examples describe some of the workshops ran during the 2011-2012 academic session at the University of Manitoba.

\subsection{LaTeX Workshop}

The LaTeX workshop was offered three times (Oct. 13, 2011, Feb. 15, 2011, and Jan 25, 2012) aimed at students completing their capstone projects and graduate thesis. The objectives were to expose students to the power of the typesetting using LaTeX through many short examples. Two instructors delivered the material using two projectors. On one screen, one presenter showed slides (created in LaTeX using the Beamer class) that highlighted key principles. The second presenter used a screen to perform live demonstrations using TeXnicCenter [7]. The examples purposely introduced errors to both the slides and examples in order to demonstrate different debugging techniques.

Since there are many best practice rules with LaTeX, but no single correct way of solving problems, the two instructors with two screens help demonstrate different ways to address the same problem. In addition, since not everything can be taught in a short workshop, the instructors purposely introduce a question that they do not 
have an answer to and must search for a solution. This provides background into using packages and reading the documentation on how to use the options to format a document.

\subsection{Orbital Mechanics using STK}

The Orbital Mechanics workshop using the Satellite Toolkit [6] took place on July 14, 2012. It was driven by UMSATS' students participating in the Canadian Satellite Design Challenge to differenciate different types of orbits (i.e., low-Earth-orbit, medium-Earth-orbits, geosynchronous orbits, Sun-synchronous orbits, and Molynia orbits). Each type of orbit was simulated using STK and using the University of Manitoba Satellite Ground Station [8] to estimate contact times.

To achieve the objectives, the instructor began with a short lecture on orbital elements and then demonstrated ranges for each parameter to form the different types of orbits using STK. To assist the learning process, hand props (i.e., inflatable globe shown and a flashlight as shown on Fig. 1) were used to reinforce the virtual simulations.

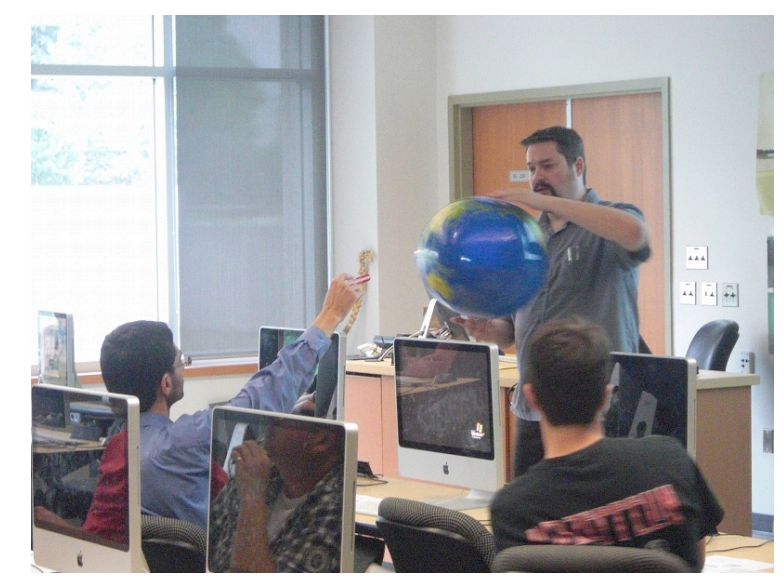

Fig. 1. STK workshop.

\subsection{Embedded Systems Workshop Series}

The Embedded Systems Workshop series are in high demand at the University of Manitoba as they attract a very wide audience that includes undergraduates, graduates, high school students, high school teachers, recent graduates, hobbyists, and industry professionals. Each series consists of beginners, intermediate, and advanced workshop held on different dates. Approximately $75 \%$ of the attendees come to all three and a small group of students attend only some of the workshops. The first series ran in the Fall of 2011 using the MSP430 LaunchPad from Texas Instruments [4] and introduced low level hardware and software techniques for working with an embedded system. The Winter 2012 series used the Arduino UNO [5] and aimed at higher level applications by taking advantage of open source and commercially available tools that enable more advanced applications to be designed and implemented in a short amount of time.

In the MSP430 series, attendees learned to used Input/Output $(\mathrm{I} / \mathrm{O})$ ports during the first workshop, then added some analog-to-digital converters, push buttons and debouching techniques, and capacitive touch interfaces as they progressed through the workshops. To make students more independent, the intermediate and advanced workshops presented a problem (i.e., using a capacitive touch sensor as shown in Fig. 2) and then guided students to solve the problem by opening user manuals and datasheets on the projector to highlight how each option was selected and configured. This portion received very high praised from attendees as often professors assume that students know how to find and interpret this information on their own without any prior explanation.

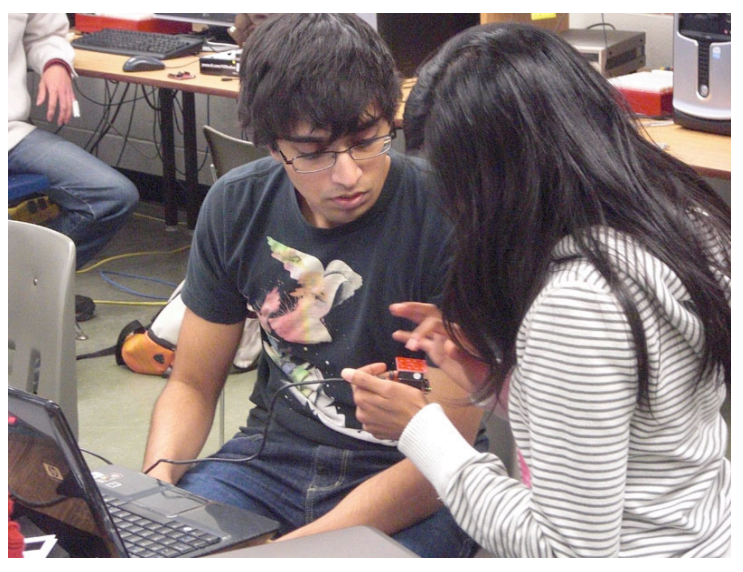

Fig. 2. Students using the MSP430 LaunchPad with a Capacitive Touch Booster Pack.

The Arduino UNO series had a more interdisciplinary flavour with students from Mechanical \& Manufacturing Engineering, Physics, and other departments who were interested in working with microcontrollers became engaged due to the popularity, reduced cost, and perceived usability of the tools. Once again, the beginners workshop focused on I/O ports, while analog-to-digital sampling and conversion, and using external memories was the focus of the intermediate workshop. For the advanced workshop, eight different Arduino Shields were purchased to explore data logging, GPS tracking, wireless interfacing, Ethernet applications, music players, screens, motors, and other areas. The instructor provided a generic background theory on interfacing different types of loads and using libraries, and then distributed different shields to each attendee to experiment in the lab for the remaining hour and a half. This forced students to read sources online, download libraries for each Shield, and interface 
them, while the instructor and assistants walked around the room offering advice and tips on what to test and how. Although the workshop was not very structured, it encouraged independence and experimentation on part of the students thus reinforcing the lifelong learning objectives.

\section{CONCLUDING REMARKS}

There are many benefits to student-run workshops. The attendees gain extensive hands-on exposure to different tools and techniques that they can used both in their studies and future careers. At the same time, the workshops provide a great networking opportunity that helps recruit students for student groups, while also introducing beginners to some of the more experienced students on campus that can serve as a resource for future projects. Just as important, the students are encouraged to play with different tools and test them on personal projects that foster life-long learning skills.

The student-instructors get a chance to practice their communication skills to deliver the presentation as well as prepare the necessary material. The students get to reinforce their knowledge and in many cases expand their understanding by preparing for and answering questions. Furthermore, the live troubleshooting serves as a great test of a student's understanding of the subject. Furthermore, those students leaning towards academia, the workshops provide valuable teaching experience, while those going into industry get to practice communicating difficult ideas to a wide range of audiences. The instructor leading the workshop gets to practice their leadership skills coordinating the team of student volunteers participating in the activities. At the same time, these students get to network and meet students that they can then recruit for various student projects such as the triple pico-satellite (T-Sat) project managed by UMSATS [9].

Overall, the student-run workshops serve to complement the classroom experience. More than 250 students in more than 20 workshops have been exposed to different tools and techniques that can help their course work and careers in industry. Since students get to run the workshops, they serve to encourage activism within the student body that further promotes more students to get involved, learn new skills, and perfect the skills to run more workshops.

\section{Acknowledgements}

Special thanks to the Department of Electrical and Computer Engineering at the University of Manitoba for their assistance with the workshops. Thanks to the IEEE Winnipeg Section for their financial support, help promoting the workshops, and feedback on activities. Finally, thanks to Shell Canada for their financial support that enabled the latest series of Embedded Systems Workshops.

\section{References}

[1] Witold Kinsner, M. G. (Ron) Britton, and Jeff Cieszecki, "Teaching teachers: Could I design, build, and test a tiny smart robot?" in Proc. of Can. Engineering Education Conf., CEEC11 (St. John's, NL: June 6-8, 2011), 2011.

[2] Dario Schor, Emily Bashford, Ryan Kehrer, Scott McKay, Mohammadreza Fazel Darbandi, Brady Russell, Chad Stasiuk, Kane Anderson, Brendan Cade, Pawel Glowacki, Benyamin Arzhangmofrad, Aaron Yanofsky, Matthew Woelk, Cody Friesen, Stephanie Reid, and Witold Kinsner, "Selected approaches to space-related educational outreach," in Proc. of Canadian Space Summit 2011, CSS11 (Calgary, AB: November 23-25, 2011), 2011.

[3] Dario Schor, Kane Anderson, Mohammadreza FazelDarbandi, Greg Linton, Matthew Woelk, Cody Friesen, Scott McKay, Katriana Soriano, Chad Stasiuk, Johnson Vilayvanh, Brendan Cade, David Terrazas, Troy Denton, Stephanie Reid, Witold Kinsner, M. D. (Ron) Britton, Dave Weichel, Debbie Leiter, Rob Streimer, Norman Lee, "Preuniversity outreach through a satellite design competition," in Proc. of Canadian Engineering Education Association Conf., CEEA12 (Winnipeg, MB: June 17-20, 2012), 2012, pp. 1-6.

[4] Texas Instruments, MSP430 LaunchPad, 2012. Available as of May 2012 from http://e2e.ti.com/group/msp430launchpad/w/default.aspx

[5] Arduino, 2012. Available as of May 2012 from http://arduino.cc/

[6] Satellite Toolkit (STK), Analytical Graphics Inc. Available as of May 2012 from http://www.agi.com

[7] TeXnicCenter. Available as of May 2012 from http://texniccenter.org

[8] Dario Schor, Witold Kinsner, and Alan Thoren, "Satellite ground station emulator: An architecture and implementation proposal," in Proc. of the IEEE Canadian Conference on Electrical and Computer Engineering, CCECE 2009, (St. John's, NL; May 3-6, 2009), pp. 874879, 2009.

[9] Dario Schor, Kane Anderson, Arash Fazel-Darbandi, Morgan May, Kris Goodmanson, Brady Russell, Brendan Cade, Mohammadreza Fazel Darbandi, Greg Linton, Matthew Woelk, Anthony Schoenfeld, Cody Friesen, Scott McKay, Witold Kinsner, M.D.(Ron) Britton, Malcolm Symonds, Diane Kotelko, Philip Ferguson, Raymond Harris, Zeev Shoichet, Walter Czyrnyj, Samuel Kovnats, and Alan Thoren, "Critical design phase of the T-Sat1 mission," in Proc. 16th CASI Astronautics Conf., ASTRO12, (Quebec City; April 24-26, 2012). 